\title{
Espaces urbains, construction identitaire et art contemporain indien : le cas des réalisations de Nikhil Chopra et de Zarina Bhimji
}

\section{Urban Spaces, Identity and Contemporary Indian Art: The Works of Nikhil Chopra and Zarina Bhimji}

Nicolas Nercam [nicolas.nercam@free.fr]

Université Michel de Montaigne de Bordeaux, France

\begin{abstract}
RÉSUMÉ
Cette communication interroge les productions de deux artistes, l'un Indien, l'autre d'origine indienne : Nilhil Chopra et Zarina Bhimji. Dans une Inde où l'appartenance communautaire reste un facteur déterminant dans la construction de l'individu, ces artistes bâtissent des correspondances entre les structures des lieux et lélaboration de l'identité individuelle. Nikhil Chopra, par ses performances, interroge tout autant les différentes facettes de son identité (coloniale en particulier) que celles des villes à l'intérieur desquelles il déambule. Zarina Bhimji place sa situation diasporique au cœur de son travail et tisse des liens entre ses différentes cultures. Les représentations du traumatisme du déracinement sont des figures récurrentes dans son travail filmique. En questionnant l'espace urbain, ces artistes prennent à défaut les définitions homogènes, de "l'individu », de la «culture » et de la « communauté ». Les entités culturelles ne sont jamais données, elles sont à construire et se doivent d’être réinterprétées.
\end{abstract}

\section{MoTS CLÉS}

art contemporain ; art de la diaspora ; arts plastiques ; art vidéo ; études postcoloniales ; histoire de l’art ; modernités artistiques extra-occidentales; performances

\begin{abstract}
This communication focuses on the study of the production of two artists (one Indian, the other of Indian descends): Nikhil Chopra and Zarina Bhimji. In India, where the communal affiliation is important for the construction of the self, both artists make connections between the urban place and the individual identity. Nikhil Chopra questions the different faces of his own identity (in particular his colonial background) as well as the multiple identities of the cities, inside of which he performed. Zarina Bhimji puts her diaspora conditions in the center of her artistic production. She ties relationship between her different cultures. The representations of the trauma of the exodus are recurring figures in her films. By interrogating the urban spaces, these two artists challenge the boundaries of the fixed notions of « individual », "culture » and « community». The cultural entities are never given but should be built and reinterpreted.
\end{abstract}

\section{KeYwORDS}

contemporary art; art of the diaspora; visual arts; art video; postcolonial studies; art history; non-western artistic modernity; performance 
La reconnaissance par la scène internationale de l'art de la production artistique indienne contemporaine date du début de ce siècle et nombre d'expositions qualifiées de "multiculturelles $^{1}$ », en Occident comme dans le reste du monde, en témoignent. Pour nous limiter ici aux manifestations les plus significatives, citons : Century City, Art and Culture in the Modern Metropolis (Tate Gallery, 2001), Edge of Desire, Recent Art in India (Asia Society, 2004-2005), Indian Highway (Serpentine Art Gallery, 2008), Chalo ! India (Mori Art Museum, 2008-2009), The Empire Strikes Back, Indian Art Today (Saatchi Art Gallery, 2009), Paris, Delhi, Bombay (C. G. Pompidou, 2011). Parallèlement à ce phénomène, la présentation d'un art contemporain diasporique, provenant principalement d'une immigration du Sud dans les pays occidentaux, voit également le jour. Parmi les expositions majeures qui engageaient la communauté indienne, relevons : Other Story (Hayward Gallery, 1989), Crossing Black Waters (City Art Gallery, 1992), Black People and the British flag (City Art Gallery, 1993, autour de l'œuvre d'Eddie Chambers), Unpacking Europe (Museum Boijmans Van Beuningen, 2001).

Ces deux veines de la production artistique contemporaine, longtemps minimisées, voir occultées au sein d'une histoire de l'art moderne et contemporain officielle ou canonique semblent dorénavant gagner en reconnaissance. Et c'est logiquement qu'au sein des grandes manifestations artistiques, les questions liées au développement de la ville à l'époque postmoderne, aux flux migratoires et à leurs répercussions sociales et culturelles, de même que les interrogations autour des concepts « d'identité postcoloniale » et d' «identité transnationale » deviennent les axes privilégiés dans la présentation et les analyses des productions artistiques modernes et contemporaines extra-occidentales et diasporiques.

Les mutations économiques, politiques, culturelles et artistiques des pays comme la Chine, le Brésil ou l'Inde trouvent leur expression la plus marquante dans le développement phénoménal des centres urbains ${ }^{2}$. Plus que jamais, l'urbaniste et l'architecte, le journaliste et le romancier, le militant politique ou associatif tout comme l'artiste plasticien deviennent, dans les modes d'expression qui leur sont propres, les concepteurs, les acteurs, les témoins et les analystes de cette extraordinaire et spectaculaire expansion urbaine. De plus, chacun tente de cerner la place qui pourrait être la sienne ou celle plus enviable qu'il souhaiterait avoir. Les grandes agglomérations indiennes n’échappent pas à ces multiples représentations dans lesquelles se mélangent intimement les images de la modernité, du chaos, du progrès, de la promiscuité, du cosmopolitisme, de l'exploitation sauvage, du plaisir extrême et de manière générale de l'opulence et de la misère et qui s'interrogent sur la place encore accordée à la personne humaine.

1 Soulignons les expositions « multiculturelles " pionnières que furent : Primitivism in 20th Century Art : Affinity of the Tribal and the Modern, organisée par William Rubin, à New York en 1984, Magiciens de la Terre, de Jean Hubert Martin, à Paris, en 1989, puis Trade Routes, organisée par Okwui Enwezor, à Johannesburg, en 1997. De nos jours, le terme « cosmopolitisme » est l'objet de nombreux débats et polémiques. D’un côté les partisans d'une lecture progressiste et émancipatrice du cosmopolitisme considèrent ce dernier comme un creuset, où les minorités sans voix trouveraient à s'exprimer (le cosmopolitisme est le lieu de formation des «identités transnationales » de la mondialisation). De l'autre, certains approchent le cosmopolitisme, non pas comme un espace de l'émancipation et de la reconnaissance des différences, mais bien comme un outil de modélisation supplémentaire à la disposition de la mondialisation capitaliste afin de transformer tous citoyens en consommateurs planétaires.

2 Inde du Nord, les mots de la ville, Cahier nº Les mots de la ville, sous la direction de Jean-Luc Racine, Maison Méditerranéenne des Sciences de l'Homme, CNRS, décembre 1999, Paris. 
Cette communication se propose d'interroger la production de deux artistes à la carrière internationale : le performeur Nikhil Chopra et la photographe/vidéaste Zarina Bhimji. Tous deux sont de famille indienne, mais Zarina Bhimji devenue citoyenne britannique, est née au sein de la diaspora indienne. Leurs réalisations combinent la double interrogation que nous venons d'évoquer : sur la ville (et les histoires qui s'y trouvent sédimentées) et sur l'identité (en abordant en particulier la question du récit autobiographique). Les deux artistes désirent, chacun à sa manière, mettre en lumière des histoires non dites, "des histoires muettes " pour reprendre l'expression de Gayatri Chakravorty Spivak, tant dans la narration des espaces urbains que dans le récit des histoires humaines (Chakravorty Spivak 1987).

\section{Nikhil Chopra, une histoire postcoloniale}

La production artistique du performeur Nikhil Chopra, né en 1974 à Kolkata, se situe à la croisée de plusieurs champs d'activité artistique : théâtre, dessin, peinture, photographie, vidéos et installation. Au Bengale, Nikhil Chopra reçu une éducation artistique en grande partie fondée sur les principes illusionnistes de la perspective linéaire albertienne. Née en Italie, cette dernière a eu, en raison des échanges commerciaux depuis la mondialisation ibérique du XVe siècle, une destinée planétaire (Belting 2012). Nous verrons ultérieurement que l'artiste saura se souvenir de sa formation première.

Encore étudiant à la Maharaja Sayajirao University de Baroda, dans l'état indien du Gujarat, Nikhil Chopra manifesta son intérêt pour le théâtre. Ce fut à partir de 2002 que l'artiste s'engagea résolument dans un travail de performances, suite à son séjour au Maryland Institue College of Art, dans l'état de l'Ohio. Dans ses performances, Nikhil Chopra incarne, maquillage et costumes à l'appui, un certain nombre de personnages faisant souvent figure d'alter ego. Ces personnages paraissent appartenir à l'histoire ancienne et coloniale de l'Inde et doivent leur existence aux souvenirs personnels, aux photographies de famille, ainsi qu'à l'imagination de l'artiste.

La première de ses créations fut le personnage de Sir Raja qui a apparu dès 2002. Sir Raja, sorte de haut dignitaire indien du XIX ${ }^{\mathrm{e}}$ siècle, sorti tout droit des écrits de Rudyard Kipling, incarne une noblesse victorienne indienne anglicisée ayant adopté les rites sociaux et le goût esthétique des dirigeants britanniques de l'Inde de l'époque. En 2003, lors de la performance intitulée Sir Raja II, dans une galerie de la ville de Columbus dans l'Ohio, le spectateur était le témoin d'un fragment de la vie fictionnelle de Sir Raja. Alors même que les spectateurs peuvent s'approcher très près de lui, Nikhil Chopra n'interagit que très rarement avec eux. Il maintient ainsi une certaine distance, une tension palpable. Il réalise, par des mouvements ritualisés, des actions de la vie quotidienne : dormir, manger, se laver, se raser, se maquiller, s'habiller, dessiner, dormir, le tout face au public. Lors de ces performances, qui peuvent durer d'une journée à plus de quatre jours, Nikhil Chopra réalise un ou plusieurs dessins au fusain ou aux pastels sur de très grands formats. Avec le personnage de Sir Raja, les poses qu'adopte l'artiste sont explicitement empruntées aux portraits d'apparat, peints ou photographiques, de l'ère victorienne. Ainsi cette réactivation du protocole victorien incite le spectateur à confronter l'histoire du British Raj à ses persistances dans la vie contemporaine. La mise en scène du personnage, reprenant les poncifs du «maharaja anglicisé », renvoie aux origines sociales et culturelles de l'artiste. Nikhil 
Chopra est issue d'une puissante famille de propriétaires terriens indiens dont la richesse, au $\mathrm{XIX}^{\mathrm{e}}$ siècle, fut étroitement liée au pouvoir colonial.

Ce fut à partir de 2005, que l'artiste décida de faire vivre le personnage de Sir Raja hors les murs de la galerie, ou de tout autre lieu culturel. Dès lors, l'inscription de ses performances dans le tissu urbain devient une composante essentielle de la construction identitaire problématisée par la démarche artistique de Nikhil Chopra. Il déclara à ce sujet : Mon travail est spécifique à chaque site. Il poursuit :

Les lumières de la ville, l'architecture, le paysage, le temps qu'il fait, la commotion et le chaos de la vie contemporaine métropolitaine, le stress et le traumatisme physique et mental, les articulations entre les histoires culturelles collectives et les mémoires individuelles, tout arrive ensemble dans la gestalt qui créé l’espace de la performance. (2010:159)

En 2005, la performance intitulée Sir Raja III visits Spitafields Market, fait déambuler le personnage dans les rues de Londres. Cette flânerie introduit dans la réalité de la rue, un fugace espace au temps décalé, une sorte d'interstice où l'artiste interroge à la fois son identité et ses origines culturelles coloniales, autant que l'identité de la ville de Londres qui doit tant à la richesse accaparée et au travail des émigrés provenant de l'ancien empire colonial.

A partir de 2007, Nikhil Chopra intensifie la dialectique entre identité individuelle et tissu urbain par la création d'un nouveau personnage, qu'il baptisa Yog Raj Chitrakar³. "Chitrakar » est le nom traditionnel que l'on attribue aux peintres ménestrels des campagnes bengalies. Littéralement, il signifie en bengali « créateur d'images ». Dans l’œuvre de Nikhil Chopra, le personnage de Yog Raj Chitrakar est une figure librement inspirée du grand-père de l'artiste, Yog Raj Chopra. Ce dernier était un riche propriétaire terrien de la vallée du Punjab et artiste paysagiste. Yog Raj Chopra fut formé à la peinture de plein air au Goldsmith College of Art de Londres, dans les années 1930. De retour en Inde, il consacra sa carrière d'artiste à peindre les paysages de la vallée du Cachemire, dans une veine esthétique naturaliste, inspirée des œuvres de John Constable et de lécole de Barbizon. A propos du personnage de Yog Raj Chitrakar, Nikhil Chopra déclara :

Yog Chitrakar est un personnage oublié du siècle victorien, il est un peintre de paysage qui part en expédition, comme un explorateur, réalisant des sortes de chroniques du monde dans lequel nous vivons. Il utilise le dessin pour transmettre ce qu'il voit. $(2009: 230)$

Le personnage de Yog Raj Chitrakar conserve de Sir Raja un aspect aristocratique de dandy victorien, mais il est plus complexe au niveau des ses identités et peut incarner différents personnages. Lors d'une même performance, il peut être : explorateur, cartographe, soldat ou prisonnier de guerre... et même parvenir, par le truchement du maquillage et du costume, à incarner des figures féminines à l'apparence de lady victorienne. Avec Yog Raj Chitrakar, le performer organise à partir d'un lieu spécifique (galerie d'art, théâtre, manufacture abandon-

3 En 2005, la performance The Death of Sir Raja III, à Mumbai, marqua la fin définitive du personnage de Sir Raja. 
née...), point de départ de sa performance, une sorte de déambulation dans l'espace urbain ${ }^{4}$. L'une de ses premières interventions, Yog Raj Chitrakar visits Lal Chowk, se tint en novembre 2007, dans l'état du Jammu-et-Cachemire indien, terre de ses ancêtres, dans la ville de Srinagar. Sur Lal Chowk (littéralement la place rouge) au cœur de la ville, l'artiste, suivit d'une cohorte d'étudiant munis de caméras, réalisa un dessin de la tour de l'horloge (monument repère de la ville de Srinagar) directement au fusain sur la chaussée. La place de Lal Chowk est un lieu sensible de la ville de Srinagar. Dans l'histoire contemporaine du Cachemire, elle fut le lieu de manifestations des opposants déterminés à la présence indienne au Cachemire et des partisans de l'indépendance de la région.

Plus récemment, en janvier 2010, avec Yog Raj Chitrakar : Memory Drawing X, Nikhil Chopra fit exécuter un trajet d'une trentaine de kilomètres à son personnage, du nord au sud de Bombay (aujourd'hui Mumbai). Cette déambulation commença, à cinq heures de l'après-midi, devant son atelier, dans le site de Bandra ${ }^{5}$. Tête rasée, arborant un costume suggérant la tenue d'un touriste muni d'un sac à dos (contenant des vivres, de l'eau, un sac de couchage, des fusains et une grande toile), Chopa entama son périple vers le sud de la ville. Ainsi accoutré, Yog Raj Chitrakar effectua, pour dessiner, plusieurs pauses sur son chemin. En particulier, il réalisa sur sa toile de grands dessins panoramiques de deux sites connus de Bombay, à partir de points de vue choisis par l'artiste : le premier, la plage de Chowpatty et Marine Drive, où s'agite la foule des touristes et des joggers, à partir du sommet des jardins suspendus qui la dominent ; le second, l'Oval Maidan de Colaba donnant sur le quartier d'affaires du sud de la ville, à partir du terrain de cricket. L'Oval Maidan est un large espace vert construit à l'époque coloniale, dominé par des édifices néo-palladiens et néogothiques des $\mathrm{XIX}^{\mathrm{e}}$ et $\mathrm{XX}^{\mathrm{e}}$ siècles. En privilégiant ces points de vue sur la ville, Memory Drawing $X$ interroge l'histoire du tissu urbain de la cité coloniale de Bombay et nous invite à une introspection sur la stratification des significations des lieux urbains. A l'époque héroïque du combat pour l'indépendance, la ville coloniale inspirait à l'élite indienne nationaliste une attitude ambivalente ; Calcutta, Madras ou Bombay étaient des lieux à la fois de sujétion et d'émancipation. L'arrivée de Gandhi sur l'échiquier politique contribua à bousculer l'attitude de l'élite anglicisée à l'égard de la ville coloniale. Le Mahatma, à l'appui des thèses défendues par Rabindranath Tagore (1919), condamna la ville, marquée du sceau de l'occidentalisation et d'un cosmopolitisme décadent, et lui opposa une image romantique (d'inspiration occidentale) du village indien, lieu où une Inde "pure, authentique et vertueuse » avait pu se perpétuer. Gandhi développa une politique de la ville qui tournait en ridicule les règles coloniales du comportement public. Dans cette optique, il inventa une tenue vestimentaire d'esprit villageois qu'il porta dans les espaces publics du British Raj. Une brèche était alors ouverte dans l'inviolabilité amidonnée de la pompe impériale britannique. Mais, le Mahatma proposa surtout aux Indiens de nouvelles manières de prendre possession des espaces publics du Raj et d'y agir. Les meetings publics et les manifestations de masses étaient de véritables nouveautés dans le paysage des villes coloniales indiennes de l'époque. En effet, à la fin du XIX siècle, les classes moyennes urbaines se réunissaient dans des "havres de sécurité coloniale ",

$4 \quad$ Avec la série de performances intitulées Memory Drawing, Nikhil Chopra fait voyagé son personnage dans les villes d'Oslo, de Tokyo, de Yokohama, de Bruxelles, de Venise, de New York, de Chicago, de Londres, de Manchester.

5 Zone de très anciennes implantations (musulmanes et chrétiennes en particulier), au nord de Mumbai. 
comme le club house, le théâtre, la mairie ou l'amphithéâtre universitaire. Gandhi redéfinit radicalement la réunion publique pour en faire une manifestation populaire de masse, au cœur même des lieux de pouvoir et de décision que sont les villes. A l'instar des métropoles occidentales, les vastes espaces publics de la ville coloniale indienne (le square, l'avenue, le gazon du maidan, l'hippodrome, le terrain de sport, le cricket field,...) devenaient les lieux essentiels de la réunion et de la contestation publiques (Chattopadhyay 2006).

Lune des dernières performances de Nikhil Chopra, Inside Out, se tint parmi les tours de la ville de Toscane de San Gimignano, en avril 2012 (Alliata Nobili 2012). Comme à son habitude, l'artiste va jouer sur les interactions entre son intervention in situ et les identités des lieux où il intervient. Avec Inside Out, le performeur indien explore les relations spécifiques qu'il entretient, depuis sa jeunesse, avec l'art de la Renaissance italienne. Cette performance débuta lorsque Nikhil Chopra prit ses quartiers à l'Arco dei Becci, une annexe de la Galleria Continua (galerie d'art de San Gimignano) lui servant de « quartier général ». Sous le regard du public, avec des gestes toujours lents et ritualisés, l'artiste préparait méticuleusement sa sortie hors de la galerie. Les costumes qu'il adopta étaient directement inspirés des fresques du $\mathrm{XV}^{\mathrm{e}}$ siècle de Benozzo Gozzoli, conservées à léglise Sant' Agostino de San Gimignano, relatant la vie de Saint Augustin. Pendant quatre jours, toujours costumé, Nikhil Chopra circulera dans les rues de la ville et de sa proche périphérie en réalisant dessins et peintures. Le premier jour, il se déguisa en berger, puis il revêtit le costume d'un artiste peintre, vêtement directement inspiré de l'autoportrait de Benozzo Gozzoli réalisé sur la fresque. Sous les traits du peintre paysagiste (nouveau clin d’œil à Yog Raj Chopra), Nikhil Chopra gravit une colline lui permettant d'avoir un point de vue panoramique sur la ville. Puis, sur sa « toile-manteau » de soie il peignit, d'après observation, les tours de San Gimignano. Enfin il circula dans les rues de la ville, la tête rasée, le visage blanchi, habillé d'un simple pagne, portant sur lui, à la manière d'une cape, sa « toile-manteau " peinte. A la fin de la performance, sur les murs de l'Arco Becci étaient présentés ses dessins de paysages, à la sépia et aux fusains. Sur les cimaises étaient également accrochés des portraits dessinés, parmi lesquels le portrait de son grand-père. Sur une table étaient encore éparpillés des reliefs d’un repas, sur le sol étaient disposés les costumes, dans le coin de la salle un matelas et à ses côtés un tas de cheveux. Inside Out joue sur l'idée de croisements des frontières entre l'histoire et le présent, entre la réalité et l'illusion, entre les cultures orientales et occidentales.

Les performances de Nikhil Chopra, à Srinagar autant qu’à Mumbai, ou sur un plan légèrement différent, a San Gimignano mettent en évidence la complexité et les difficultés de la lecture des lieux urbains mêlant les strates liées à l'histoire coloniale à celles des histoires contemporaines. Les significations multiples des lieux trouvent leur reflet dans l'identité stratifiée et changeante de l'artiste qui revendique pleinement ses appartenances culturelles composites et en particulier « la digestion » de son patrimoine colonial. L’ouvre de Nikhil Chopra témoigne d'une subjectivité postcoloniale particulièrement complexe. Elle pourrait illustrer la notion " d'individu colonisé » développée par Edward W. Saïd qui déclarait : « L'identité du colonisé est donc plurielle, elle rassemble de multiples types différents, [...], dans de multiples lieux distincts, à diverses époques » $(2008: 412)$. 


\section{Zarina Bhimji, une histoire de l'exil}

Le travail de la photographe et vidéaste Zarina Bhimji, régulièrement présenté lors de grandes manifestations multiculturelles (biennales de Venise, de Sao Paulo, d'Istanbul, Documenta de Kassel...), bénéficie, de nos jours, d'une aura internationale. Zarina Bhimji place sa situation diasporique spécifique au cœur de son travail artistique qui tisse des liens entre les cultures indiennes, africaines et britanniques dont il se nourrit. L’extraordinaire et tragique odyssée, à la fois coloniale et postcoloniale, de Zarina et de sa famille permet d'en éclairer la genèse.

Zarina Bhimji est née en Ouganda, en 1963 dans la ville de Mbarara, de parents d’origine indienne. La présence d'une importante communauté indienne en Ouganda est le fruit de la politique coloniale de l'Empire britannique. A partir de 1896, la British East Africa Company lança un vaste chantier pour la construction d'une voix de chemin de fer nécessitant la venue d'une importante main-d’ouvre immigrée, provenant principalement du sous-continent indien. Dès le début du $\mathrm{XX}^{\mathrm{e}}$ siècle, la population indienne déjà bien intégrée (ouvriers, marchands, commerçants) constituait une part significative de la société ougandaise. L'indépendance du pays fut acquise en 1962 et en 1971 le général Idi Amin Dada prend le pouvoir suite à un coup d'état. Il désire construire une « Nation africaine noire », en restituant aux « authentiques africains » le pouvoir que le système colonial avait spolié. Dans le cadre de cette politique, en août 1972, le général exige le départ, dans un délai de trois mois, de toute la population asiatique du pays. Plus de 50000 personnes sont invitées, manu militari, à quitter l'Ouganda. La famille Bhimji, dans un premier temps, décide de rester en Ouganda, vivant alors dans une semi-clandestinité. Mais la guerre civile, orchestrée par Idi Amin Dada, est d'une telle violence que la famille (détentrice de passeports britanniques) décide de quitter définitivement l'Ouganda pour la Grande Bretagne, en 1974. L'arrivée en Angleterre fut difficile. Dans les années 1970, nombre d'immigrants indo-pakistanais d'Ouganda, arrivant en Grande Bretagne par les aéroports de Londres ou de Manchester, furent accueillis par des militants du National Front et leurs slogans xénophobes mettant en garde la population britannique " de souche » des dangers d'une « immigration de couleurs " (Humphry \& Ward 1974). Une fois installée, Zarina Bhimji suivra une formation artistique, successivement à la Slade Shool of Fine arts, puis au Goldsmith's College de Londres, enfin à la Leicester Polythechnic.

Comme le souligne l'historienne de l'art Tania V. Guha, la critique du « pouvoir institutionnel » fut un concept clés de la production artistique de Zarina Bhimji (Guha 2001). La véritable fascination qu'éprouve l'artiste pour cette articulation entre pouvoir et institution réside dans l'intérêt qu’elle porte aux capacités de dissémination et d’objectivation idéologiques des structures institutionnelles. En 2001, Zarina déclara à ce sujet :

Je suis obsédée par le dévoilement des relations qui lient les institutions au pouvoir, ainsi que par l'exposition du rôle des institutions dans la dissémination des idéologies. Je veux comprendre comment tout cela fonctionne [...]. Je crois que comprendre un système vous permet de mieux l'infiltrer. (Bhimji 2001 : 92)

Dans nombre des installations qu'elle propose depuis plus de vingt ans, l'artiste s'attaque aux institutions muséales, comme aux structures universitaires et aux organisations hospitalières. En 1992, à l'Ikon Gallery de Birmingham, Zarina Bhimji créé l'installation I will always be here, 
composée de soixante boites en carton, recouvertes d'une vitre. Chaque boite contenait des objets épars : morceaux de tissus, de cheveux, des cendres, des dents, des photographies... En faisant écho à la classification ethnographique et muséale des objets culturels, les alignements de ces boites, tel un alignement de cercueils, renvoyaient au caractère mortifère de l'institution muséale. Ce travail constituait le point dorgue des recherches de Zarina Bhimji, entreprises, depuis 1989, au Southeast Asian Department du Victoria \& Albert Museum de Londres. Dans ses réserves, le musée disposait de boites à l'intérieur desquelles étaient conservés et classés différents objets. La présence de ces boîtes en carton (véritables boîtes à chaussures) dépassait le cadre de l'institution du musée, pour élargir la critique à tous types de système de classification et de normalisation. Ainsi, la classification policière était également visée. Lors d'une discussion avec un policier, ce dernier l'informa que les preuves lors d'une exaction étaient très souvent réunies dans une boite à chaussure.

Une problématique similaire constituait le fil conducteur de l'installation photographique 1822-Now, présentée pour la première fois au MuKHA Museum d'Anvers, en 1993. Des portraits photographiques de personnes de différentes origines raciales étaient présentés sur des étagères d'une sorte de bibliothèque. Cette installation faisait explicitement allusion aux classifications raciales du XIX ${ }^{e}$ siècle, érigées au rang de science et ayant servies de caution à l'expansion coloniale européenne. « 1822 » fait probablement référence à l’année de naissance du scientifique britannique Francis Galton (1822-1911), fondateur de l'eugénisme. Galton entreprit une traduction des différences sociales sur un strict plan biologique. Ainsi, leugénisme refusait toute mixité sociale car cette dernière entrainait une disparition des caractéristiques les plus hautes (portées par les strates les plus élevées de la société occidentale) de la race humaine. L'eugénisme vint alimenter les théories évolutionnistes qui triomphèrent à la fin du XIX ${ }^{e}$ siècle. Ces théories considéraient les sociétés extra-occidentales comme des témoins fossiles des stades de l'humanité. Ainsi, les «naturels » du XVIII siècle devenaient les " primitifs » au XIX ${ }^{e}$ siècle et le mot se chargea d'un sens péjoratif que tout oppose au « civilisé ». Au travers des expositions universelles et coloniales l'Europe met en scène "l'Autre culturel », par des représentations aussi fausses que stéréotypées (l'exotisme ne donne à voir que la surface des choses). Ces mises en scène, reconstituant la vie quotidienne primitive, servent de faire-valoir à la richesse et aux savoirs des puissances occidentales et de prétexte à montrer que l'Europe a su tirer profit, face aux mondes «primitifs» de toutes les ressources de la terre.

Pour Zarina Bhimji, la perception occidentale de cet « Autre culturel » passerait donc toujours au travers du filtre déformant de l'eugénisme. En réaction, les portraits photographiques qu'elle présente dans 1822-Now ne se limitent pas à un cadrage serré sur des visages aux traits inexpressifs comme pour une photographie d'identité policière ou un cliché ethnographique et certains sont même totalement flous, au point de rendre toute identification du modèle impossible. Cet effacement relatif des visages se veut acte de résistance face aux techniques arbitraires et aux classifications autoritaires généralement utilisées en la circonstance. Mais il dénonce avec plus de force encore le fait que les hommes et les femmes extra-occidentaux, perçus à travers les stéréotypes du colonialisme, ne possèdent plus d'identité propre et restent comme invisibles en tant que personne humaine unique.

Le questionnement critique sur l'institution (en particulier l'institution artistique du musée et de la galerie) se trouve, depuis le début du XX ${ }^{e}$ siècle, au cœur des démarches de la modernité 
artistique (depuis l'anti-art de Dada, les ready-made de Duchamp et jusqu'à l'art social hors les murs des années 1960-1970). Les artistes postmodernes extra-occidentaux et de la diaspora du Sud installée en Europe et en Amérique du Nord, tout en poursuivant une analyse critique de l'œuvre d'art et du processus de sacralisation propre au musée, apportèrent une coloration particulière à ce questionnement ${ }^{6}$. Le musée fut également le lieu de l'objectivation " scientifique " d'une polarité entre "primitif » et "civilisé » et contribua à cristalliser les clichés sur l'altérité culturelle. Bernard S. Cohn, dans son ouvrage Colonialism and its forms of knowledge, présente les efforts des orientalistes et des africanistes britanniques à connaître les us et coutumes et les langues locales comme partie intégrante d'un vaste projet de normalisation et de contrôle des pays colonisés (Cohn 1996).

Avec Out of Blue, court-métrage de 16 minutes, Zarina Bhimji entendait témoigner des traumatismes profonds provoqués par les épreuves du déplacement et de l'exil, cette " fissure à jamais creusée entre l'être humain et sa terre natal ", comme l'écrira Edward W. Saïd (2008). Ce concept d'exil masque en fait une grande diversité de situations que l'on peut néanmoins regrouper en fonction du degré d’intentionnalité du déplacement ${ }^{7}$ sous trois intitulés : "émigré ", " expatrié » et " réfugié ». Zarina Bhimji et sa famille ont connu les trois situations, émigrés de l'Inde, expatriés de l'Ouganda et réfugiés en Angleterre. Edward W. Saïd insiste sur le sentiment de «non appartenance » qui affleurerait lorsque des individus, ou des groupes d'individus, sont brutalement déracinés. S’inscrivant dans le sillage des distinctions énoncées par Saïd, le sociologue Paul Gilroy considère que l’entité « diaspora » est définit par « une dispersion forcée et un éparpillement à contre cœur, bien plus que par une expérience du déplacement librement choisie» (1997 : 318). L'exilé tenterait toujours de se reconstituer une identité en particulier à partir des brisures et des outrages subis sur les chemins de l'exil. L'œuvre de Zarina Bhimji, hautement autobiographique, témoigne de cette quête.

Out of Blue représente différentes étapes d'une journée, de l'aube jusqu'à un départ en avion. Il fut commandité par Okwui Enwezor, pour la Documenta 11 de Kassel, en 2002. Ce fut en 1998, que Zarina revint en Ouganda et réalisa des photographies de lieux associés à l'histoire de son père. Ces clichés constitueront une sorte de repérage cinématographique pour Out of Blue et quatre ans plus tard, elle effectuera un nouveau voyage en Ouganda afin d'y réaliser son film. Le film évoque cette "journée particulière ", au travers de la représentation de paysages de l'Ouganda, principalement par des plans fixes et de lents panoramiques. Certains de ces paysages représentent une nature généreuse et verdoyante, d’autres des terres dévastées par les flammes (double allusion à la culture du brûlis et à la guerre civile). Puis se succèdent des images de rues désertées, de cimetières, de baraques d'ouvriers ou de militaires abandonnées et de cellules, aux murs lépreux, vidées de ses prisonniers. Enfin, le film se termine par des images d'un aéroport comme délaissé et par un avion prêt à décoller. Le son joue un rôle important et

6 Des artistes femmes de la diaspora comme Chila Kumari Burman, revendiquant sa double appartenance Scouse et Punjabi, réalisa en 1993, une série d’estampes, représentant un ensemble de portraits, parmi lesquelles Fly Girl Watching the World et Wild Woman between two Cultures. L'objectif de cette série était de mettre en lumière la marginalisation d'un Black Art par les instituions de promotion artistique.

7 Ainsi, d'après Edward W. Saïd, l'émigré et l'expatrié seraient plutôt caractérisés par " un déplacement volontaire ", alors que le réfugié serait caractérisé par « un déplacement forcé » pour des raisons politiques, économiques ou environnementales (Saï 2008). 
ne se limite pas à accompagner la projection des images. La bande son est composée d'une imbrication de bruits d'oiseaux, de vols de moustiques, de grondement du tonnerre, de chants, de détonations de fusils, de voix divers donnant naissance à une sorte de cacophonie harmonieuse. Parmi les voix, celle d'Idi Amin Dada, retransmis par la radio en août 1972 était reprise en boucle, à de nombreuses reprises dans le film : «J'ai donc signé un décret, devant être appliqué dès aujourd'hui 9 août 1972, révoquant tous les permis et certificats de résidence des personnes mentionnées préalablement... $»^{8}$

Out of Blue nous propose une sorte d'inventaire du legs du général Idi Amin Dada inscrit insidieusement dans le paysage contemporain de l'Ouganda. Un effet de catharsis semble être ici recherché par l'artiste. Par le traitement des images, leur montage et l'emploi du son, le spectateur est conduit à expérimenter les sentiments d'insécurité et de peur qu'ont pu éprouver ceux qui ont vécu, en Ouganda dans les années 1970, la terrible politique d’épuration du général Idi Amin Dada.

Ce film complète et prolonge l'installation intitulée She loved to Breath - Pure Silence, présentée en 1987 à la Whitechapel Art Gallery de Londres. Cette production voulait décrire avec un minimum d'éléments (des photographies, des textes et des épices) quelques uns des espaces parcourus durant son exil par la diaspora asiatique. Quatre panneaux de plexiglas suspendus au plafond constituaient sa structure centrale. Les deux premiers présentaient, plaquées sur des draps de mousseline, des photographies de cadavres d'oiseaux et de chaussures apparemment abandonnées lors d'un déplacement forcé et précipité. Cette juxtaposition rappelait les risques graves et parfois mortels encourus par les migrants dans leur fuite. Sur le troisième panneau, un texte court dressait le constat d'une discrimination que vivaient quotidiennement les immigrants indo-pakistanais en Grande Bretagne : "Each morning at 5:00 am they scrubbed the floor... Sometimes these white people on their way to work laughed at their Indianess... sucked their teeth dismissing them ». Enfin dans le dernier panneau, une paire de gants chirurgicaux en latex était posée sur un drap de mousseline. A ses côtés, un agrandissement du tampon officiel du British Home Office Visa était imprimé directement sur le drap : "The time limit on the holder's leave to enter the United Kingdom is thereby removed ». Ce document était signé et daté à l'encre bleue : 7 novembre 1975. La présence glaçante des gants de latex conférait à l'installation une connotation sinistre. Elle rappelait les tests de virginité imposés par les autorités britanniques dans les années 1970, à toutes les femmes arrivant à l'aéroport d'Heathrow et venant de l'Ouganda.

\section{Conclusion}

Zarina Bhimji et Nikhil Chopra interrogent les notions de flux migratoire, d'exil et d'inerculturalité au sein de l'espace de la ville. Ils participent ainsi à cette « narration urbaine et périurbaine postcoloniale » car, chacun à leur manière, ils considèrent que les principales caractéristiques de la mondialisation ne peuvent se comprendre qu'en relation à une histoire coloniale, contestant le principe de suprématie des centres sur les périphéries. Comme le souligne le critique et

8 Paroles d'Idi Amin Dada, repris par Katherine Stout. 
commissaire d'exposition Okwui Enwezor (Enwezor 2014) l'extension des idées postcoloniales dans le champ artistique a participé à un regain d’intérêt pour les théories de la résistance, ainsi que pour les diverses formes du cosmopolitisme (culturel et formel principalement). Dans cette veine, il est à relever le film Territories (Territoires) d'Isaac Julien présenté en 1984, sur la communauté afro-caribéenne de Noting Hill, ainsi que celui de John Akomfrah, Handsworth songs, en 1986, sur les émeutes communautaires à Londres et à Handsworth.

L'intervention artistique en milieu urbain débouche sur une conception du "lieu » qui ne doit pas être uniquement pensé en terme de " site et non-site », de "privé et public », "d'intimité et d'institutionnel », mais doit également être envisagé comme un fait social vécu au travers des expériences du déplacement, de la mobilité, de la migration, de la dépossession et de la déterritorialisation. Au sein des productions de Zarina Bhimji et de Nikhil Chopra, l'art in situ révèle une mémoire effacée d'un lieu particulier et interroge la place comme un lieu politique et social. Le lieu urbain, perçu par le discours postcolonial, constitue l'épicentre d'une mémoire effacée. Okwui Enwezor déclare :

[...] La résurgence de l'urbanisme et le recours à de multiples formes de subjectivité urbaine dans l'art contemporain constituent aujourd'hui une réflexion sur l'usage à la fois esthétique et politique du lieu en tant quélément paradigmatique de la vie actuelle. (2014:34)

A l'unisson de la pensée d'Edward Saïd (2008), Okwui Enwezor considère que l'artiste postcolonial se doit de travailler aux nouvelles formes des "identités transnationales » inscrites dans les villes mondialisées. Le géographe David Pinder, dans son article Arts of Urban exploration (2005) déclare :

Des pratiques artistiques, en relation avec les questions relatives à la spatialité urbaine des pouvoirs dominants, sont critiques et politisées lorsqu'ils perturbent notre vie quotidienne urbaine, quand ils utilisent des moyens artistiques et créatifs afin d'explorer des problèmes et des conflits sociaux sans pour autant prescrire de solutions à ces problèmes et lorsqu'ils résistent au processus par lequel les espaces urbains sont conçus comme devant servir, le plus souvent, les intérêts du capital et ceux de létat, quand ils recherchent et encouragent à plus d'alternatives démocratiques.

Edward W. Saïd considère que ce traumatisme du déplacement doit constituer, dans la conscience de l'exilé - émigré comme réfugié - un terreau fertile afin de «cultiver une subjectivité scrupuleuse, ni indulgente, ni maussade» (2008 : 254). Lorsque les identités des exilés sont formées dans une relation excentrique avec la citoyenneté que confère l'État-nation et qu'ils décident d'agir comme un " citoyen du monde » ou comme " un citoyen cosmopolite », alors ils ont le potentiel pour distancier et critiquer l'autorité symbolique de la nation (tant de leur nation d'origine que de leur nation d'accueil). Les exilés sont porteurs d'au moins deux cultures et cette pluralité de vision donne naissance à une prise de conscience de situations simultanées, comme en contre-point. Edward W. Saïd en arrive à conférer une mission politique et sociale à l'exilé, en affirmant que ce dernier peut constituer une sorte "d'alternative aux institutions de masse qui dominent le monde moderne. L'exil n'est pas, après tout, une question de choix : 
on nait en exil, ou on se retrouve en exil » (2008: 254). Les historiens de l'art et commissaires d'exposition Jean Fisher et Gerardo Mosquera abondent en ce sens en affirmant : "Si l'exilé fut la figure de la première modernité, l’immigrant le membre de la diaspora est la figure de la postmodernité avec son sujet décentré et déterritorialisé » (2005:3).

Le film de Zarina Bhimji, de même que les performances de Nikhil Chopra problématisent une double représentation historique : D'un côté l'histoire contemporaine de l'Ouganda et celle des métropoles indiennes, de l'autre l'histoire coloniale, en l'occurrence britannique. Zarina Bhimji et Nikhil Chopra combinent intimement ces deux histoires afin de comprendre la complexité d'une immigration postcoloniale.

Mais cette imbrication des significations ne se limite pas aux références historiques et sociologiques, mais touche également aux sources esthétiques mobilisées dans Out of Blue et dans Memory Drawing. Ainsi, la traduction des paysages d'Ouganda (dans des tonalités chaudes de début de journée) ne serait pas étrangère au traitement pictural des paysages de Turner (exposés à la Clore Gallery de la Tate Britain). Du côté de Nikhil Chopra, la référence à l'art du paysage occidental et à l'esthétique de la mimesis est clairement revendiquée. Les références picturales des dessins de Nikhil Chopra s'alimentent en particulier aux peintres reporters britanniques de la fin du XIX e siècle comme William \& Thomas Daniell ainsi que Hodges. De même, dans nombre de ses immenses dessins, la représentation de l'architecture néoclassique propre aux villes coloniales indiennes y est abondamment figurée (comme une sorte de motif récurent). L'architecture filmée par la caméra de Zarina Bhimji mélange savamment la représentation de bâtiments officiels de style colonial et des architectures plus vernaculaires comme les baraques des ouvriers.

L'œuvre de Zarina Bhimji tout comme celle de Nikhil Chopra s'inscrivent dans une sorte "d'espace interstitiel », pour reprendre ici une formule d'Homi K. Bhabha (1994). Cela nous invite à une interprétation de la production de ces deux artistes qui déplace les polarités de convention (Indianité / Britishness - masculin / féminin - Orient / Occident...) pour développer de multiples interfaces qui brouillent les notions considérées comme homogènes et unitaires, de « culture ", « d'identité » et « d'histoire».

\section{Références bibliographiques}

Alliata Nobili, A. (2012). San Gimignano, Galleria Continua, Nikhil Chopra, Inside Out. Artasiapacific, 80. Bhabha, H. K. (1994). The Location of Culture. New York : Routledge.

(2007). Les lieux de la culture, une théorie postcoloniale. Paris : Payot.

Belting, H. (2012). Florence et Bagdad, une histoire du regard entre Orient et Occident. Paris : Gallimard.

Chattopadhyay, S. (2006). Representing Calcutta, Modernity, Nationalism, and the Colonial uncanny. Londres : Routledge.

Cohn, B. S. (1996). Colonialism and its forms of Knowledge : the British India. New Jersey : Princeton University Pres.

Enwezor, O. (2014). Migrants, nomades, pèlerins : globalisation de l'art contemporain. In Une Histoire, art, architecture, design des années 1980 à nos jours (pp. 28-39). Paris : C. G. Pompidou. 
Gilroy P. (1997). Diaspora and the détours of Identity. In K. Woodward (Dir.), Identity and Difference. Londres : London and Tousand Oaks/Sage Publication.

Guha T. V. (2001). Face to Face : an interview with Zarina Bhimji. In A. Ghosh, \& J. Lamba (Eds.), Beyond Frontiers, Contemporary British Art by Artists of South descent (pp. 91-102). Londres : Saffron Books.

Humphry D., \& Ward, M. (1974). Passports and Politics. Londres: Penguin.

Pinder D. (2005). Arts and Urban Exploration. In Cultural Geographies (4) (pp. 383-411). New York : Sage Publication.

Racine J.-L. (Dir.) (1999). Inde du Nord, les mots de la ville, Cahier ${ }^{\circ} 4$ Les mots de la ville. Paris : Maison Méditerranéenne des Sciences de l'Homme, CNRS.

Saïd E. W. (2008). Représenter le colonisé : les interlocuteurs de l’anthropologie. In Réflexions sur l'exil et autres essais (pp. 385-412). Paris : Actes Sud.

- (2008). Réflexions sur l'exil. In Réflexions sur l'exil et autres essais (pp. 241-257). Paris : Actes Sud.

Spivak, G. C. (1987). Can the Subaltern speak? In C. Nelson, \& L. Grossberg (Eds.), Marxism and the Interpretation of Culture, (pp. 271-316). Urbana : University of Illinois Press.

- (2009). Les Subalternes peuvent-elles parler? Paris : Éditions Amsterdam.

Stout, K. (2003). Zarina Bhimji : Art Now. Londres : Tate Publishing.

Tagore, R. (1919). The Centre of Indian Culture. Calcutta : Visva-Bharati.

- (1951). A vision of India's History. Calcutta : Visva-Bharati.

\section{Catalogues d'exposition}

(2001). Unpacking Europe, Museum Boijmans Van Beuningen, Rotterdam: NAI Publishers.

(2005). Edge of Desire, Recent Art in India, Art Gallery of Western Australia, Perth, Asia Society Musuem, New York.

(2009). Yo y el otro. Retratos en la fotografia india contemporanea, édité par ARTIUM de Alava, Ayuntamineto de Barcelona, instituto de Cultura y Lunwerg.

(2010). My work is site-specific. Propos de Nikhil Chopra, catalogue de la $8^{\mathrm{e}}$ édition de The Shanghai biennale. 
